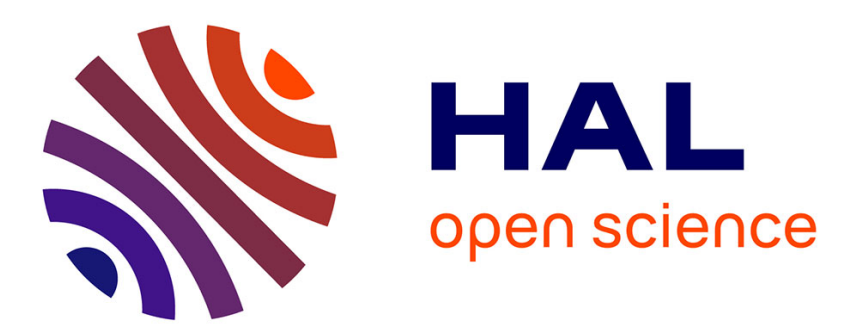

\title{
Formulation variationnelle pour le calcul de la diffraction d'une onde acoustique par une surface rigide
}

\author{
Alain Bamberger, Tuong Ha Duong
}

\section{To cite this version:}

Alain Bamberger, Tuong Ha Duong. Formulation variationnelle pour le calcul de la diffraction d'une onde acoustique par une surface rigide. Mathematical Methods in the Applied Sciences, 1986, 8 (1), pp.598-608. 10.1002/mma.1670080139 . hal-01564586

\section{HAL Id: hal-01564586 \\ https://hal.science/hal-01564586}

Submitted on 18 Jul 2017

HAL is a multi-disciplinary open access archive for the deposit and dissemination of scientific research documents, whether they are published or not. The documents may come from teaching and research institutions in France or abroad, or from public or private research centers.
L'archive ouverte pluridisciplinaire HAL, est destinée au dépôt et à la diffusion de documents scientifiques de niveau recherche, publiés ou non, émanant des établissements d'enseignement et de recherche français ou étrangers, des laboratoires publics ou privés.

\section{(c)(1)}

Distributed under a Creative Commons Attribution| 4.0 International License 


\title{
Formulation Variationnelle pour le Calcul de la Diffraction d'une Onde Acoustique par une Surface Rigide
}

\author{
A. Bamberger et T. Ha Duong, Palaiseau
}

This work is a continuation of [1]. We give a space-time variational formula to the problem of the scattered acoutic wave by a hard body, using the double layer retarded potential technique. New schemes are constructed from this variationnal formula, for which we prove the stability and errors estimates.

\section{$\$ 1 \quad$ Introduction}

Nous avons étudié dans [1] l'utilisation d'un potentiel retardé de simple couche pour calculer l'onde acoustique diffractée par une surface régulière soumise à une condition de Dirichlet. Nous avons dans ce cas obtenu une formulation variationnelle en temps et en espace de l'équation intégrale permettant de calculer la densité du potentiel cherché. Il en résulte aussi des résultats de stabilité et de convergence habituellement non traités dans les travaux numériques concernant ce problème.

Nous considérons ici le problème de Neumann:

$$
\left(\mathbf{P}_{+}\right) \begin{cases}\square u(t, x)=0 & t \in \mathbf{R}, x \in \Omega_{+} \\ u(t, x)=0 & \left(t \leqslant 0, x \in \Omega_{+}\right) \\ u_{t}(t, x)=0 & \\ \frac{\partial u}{\partial v}(t, x)=g(t, x) & (t \in \mathbf{R}, x \in \Gamma)\end{cases}
$$

où $\Omega_{+}$est l'extérieur d'un domaine borné de $\mathbf{R}^{3}$, de frontière $\Gamma$. La fonction $g(t, x)$ est nulle pour $t \leqslant 0$.

La généralisation des résultats de [1] dans ce cas est facile en utilisant une représentation de $u$ par un potentiel retardé de double couche. Nous donnons les résultats correspondants dans le $\$ 2$, où se trouve aussi une expression de la formulation variationelle qui ne contient pas d'intégrale hyper-singulière. Le $\$ 3$ est consacré à la discrétisation de cette formulation variationnelle, le $\S 4$ aux résultats de stabilité et convergence. 


\section{$\$ 2$ Le potentiel retardé de double couche}

Nous donnons dans ce paragraphe quelques résultats sur le potentiel retardé de double couche: en particulier, l'existence et l'unicité dans un cadre fonctionnel de Sobolev de la représentation de la solution du probleme $\left(\mathbf{P}_{+}\right)$par un tel potentiel, ainsi qu'une propriété de coercivité pour l'opérateur $D$ (voir (2.3)) permettant de calculer la densité de ce potentiel. Ces résultats sont obtenus grâce à une transformation de Fourier-Laplace, et sont absolument analogues à ceux déjà obtenus dans le cas du problème de Dirichlet. La différence essentielle concerne le caractère singulier de $D$, et sera traité à la fin de ce paragraphe.

\subsection{Resultat d'existence et d'unicité}

On cherche donc à résoudre le problème $\left(\mathbf{P}_{+}\right)$en cherchant une représentation de sa solution éventuelle par un potentiel retardé de la forme:

$$
\left\{\begin{aligned}
u(t, x)= & \frac{+1}{4 \pi} \int_{\Gamma} \nu_{y} \cdot \nabla_{x}\left(\frac{\varphi(t-|x-y|, y)}{|x-y|}\right) \mathrm{d} \sigma_{y} \\
= & \frac{+1}{4 \pi} \int_{\Gamma} \frac{\nu_{y} \cdot(y-x)}{|y-x|^{2}}\left\{\frac{\partial \varphi}{\partial t}(t-|x-y|, y)\right. \\
& \left.+\frac{\varphi(t-|x-y|, y)}{|x-y|}\right\} \mathrm{d} \sigma_{y}
\end{aligned}\right.
$$

pour $t>0, x \in \Omega_{+} \cdot v_{y}$ étant la normale vers $\Omega_{+}$du point $y \in \Gamma$.

ce qui revient à associer au problème $\left(\mathbf{P}_{+}\right)$un problème intérieur $\left(\mathbf{P}_{-}\right)$de même donnée frontière $\frac{\partial u}{\partial v}$. La densité $\varphi$ du potentiel (2.1) est alors:

$$
\varphi(t, x)=u_{-}(t, x)-u_{+}(t, x) \quad(t \in \mathrm{R}, x \in \Gamma) .
$$

où, de façon générale on écrit $f_{+}$la trace sur $\Gamma$ d'une fonction $f$ définie dans $\bar{\Omega}+$.

$(-)$

La même notation s'étend aux distributions quand cette trace est définie.

La formule de représentation de Kirchoff permet ensuite de calculer $\varphi$ comme solution de l'équation:

$$
D \varphi=g
$$

où pour tout $t \in \mathrm{R}, x \in \Gamma$ :

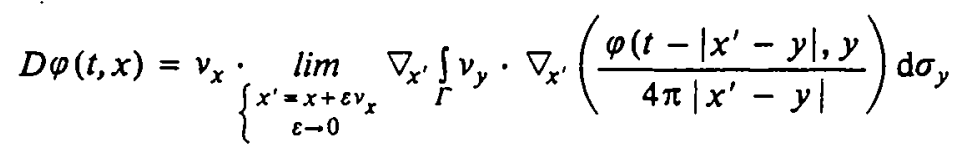

la limite est à prendre au sens de distribution, l'intégrale 


$$
\int_{\Gamma} v_{x} \cdot \nabla_{x}\left(v_{y} \cdot \nabla_{x} \frac{\varphi(t-|x-y|, y)}{|x-y|}\right) \mathrm{d} \sigma_{y}
$$

pour $x \in \Gamma$ est une intégrale hyper-singulière, avec un noyau en $11 x-\left.y\right|^{3}$. Comme dans le cas d'un potentiel de simple couche pour le problème de Dirichlet, nous étudions l'opérateur $D$ et l'équation (2.2) en passant par une transformation de Fourier-Laplace en $t$, ce qui est loisible car les fonctions considérées sont nulles pour $t \leqslant 0$.

Posant pour une telle fonction $f$, et pour $\omega \in \mathrm{C}, \operatorname{Im} \omega>0$ :

$$
\hat{f}(\omega)=\int_{-\infty}^{+\infty} \mathrm{e}^{\mathrm{i} \omega t} f(t) \mathrm{d} t
$$

on est amené à résoudre simultanément des problèmes de Neumann extérieur et intérieur pour l'équation de Helmholtz:

$$
\left(\mathbf{P}_{ \pm}^{\omega}\right) \begin{cases}\left(\Delta+\omega^{2}\right) \hat{u}=0 & \text { dans } \Omega_{ \pm} \\ \left(\frac{\partial \hat{u}}{\partial \nu}\right)_{ \pm}=\hat{g} & \operatorname{sur} \Gamma \\ \hat{u} \in \mathbf{H}^{1}\left(\Omega_{ \pm}\right) & \end{cases}
$$

cette dernière condition exprime le fait que $u$ solution de $\left(\mathbf{P}_{ \pm}\right)$est à énergie finie. La formule de Green permet de vérifier facilement l'existence et l'unicité de la solution $u$ de $\left(\mathbf{P}_{ \pm}^{\omega}\right)$, qui est alors représentée par un potentiel de surface de double couche:

$$
\hat{u}(\omega, x)=\frac{1}{4 \pi} \int \frac{\partial}{\partial \nu_{y}}\left(\frac{-e^{\mathrm{j} \omega|x-y|}}{|x-y|}\right) \hat{\varphi}(\omega, y) \mathrm{d} \sigma_{y} \quad\left(x \in \mathbf{R}^{3} \bigvee\right)
$$

où la densité $\hat{\varphi}$, égale à $\hat{u}_{-}-\hat{u}_{+}$, est aussi solution de l'équation intégrale:

$$
D_{\omega} \hat{\varphi} \equiv \frac{\partial \hat{u}}{\partial \nu}=\hat{g}
$$

avec

$$
D_{\omega} f(x)=v_{x} \cdot \nabla_{x}\left(\int \frac{\partial}{\partial v_{y}}\left(\frac{-\mathrm{e}^{\mathrm{i} \omega|x-y|}}{4 \pi|x-y|}\right) f(y) \mathrm{d} \sigma_{y}\right) \quad(x \in \Gamma) .
$$

De plus, l'examen de la dépendance en $\omega$ de $\hat{u}, \hat{\varphi}$ donne les inégalités:

$$
\left\{\begin{array}{l}
\int_{\Omega_{+} \cup \Omega_{-}}\left(|\nabla \hat{u}(\omega, x)|^{2}+|\omega \hat{u}(\omega, x)|^{2}\right) \mathrm{d} x \leqslant C|\omega \hat{g}(\omega, .)|_{-1 / 2}^{2} \\
\text { pour tout } \omega \in \operatorname{Im} \omega=\sigma_{0}>0 \\
\text { La constante } C \text { ne depend que de } \Gamma \text { de } \sigma_{0}
\end{array}\right.
$$


et

$$
\left\{\begin{array}{l}
|\phi(\omega, .)|_{1 / 2} \leqslant C|\omega \hat{g}(\omega, .)|_{-1 / 2} \\
\operatorname{Im} \omega=\sigma \geqslant \sigma_{0}>0
\end{array}\right.
$$

où l'on a noté $|f|_{s}$ pour la norme de $f \in \mathbf{H}^{s}(\Gamma)$, espace de Sobolev classique. Voir [1].

Avec la formule de Parseval, on traduit aisément tout cela en des résultats pour $u$ et sa représentation (2.1). Le cadre fonctionnel choisi est défini par les espaces de type Sobolev:

$$
H_{\sigma}^{s}\left(\mathbf{R}_{+}, \mathbf{E}\right)=\left\{f \in L^{\prime}(E) ; \quad \int_{-\infty+\mathrm{i} \sigma}^{+\infty}|\omega|^{2 s}\|\hat{f}(\omega)\|_{E}^{2} \mathrm{~d} \omega<\infty\right\}
$$

où $E$ étant un espace de Hilbert (essentiellement $\mathbf{H}^{r}(\Gamma)$ et $\mathbf{H}^{r}\left(\Omega_{ \pm}\right)$), $L^{\prime}(\mathbf{E})$ désigne les distributions sur $R$, à valeurs dans $\mathbf{E}$, nulles pour $t \leqslant 0$ et majorées à $t \rightarrow+\infty$ par une exponentielle en $t$.

On notera $|f|_{\sigma, s, r}$ pour la norme de $H_{\sigma}^{s}\left(\mathbf{R}_{+}, \mathbf{H}^{r}(\Gamma)\right)$. On a alors le

Théorème 1 a) Sous l'hypothèse

$$
g \in H_{\sigma_{0}}^{0}\left(\mathbf{R}_{+}, \mathbf{H}^{-1 / 2}(\Gamma)\right)
$$

pour un $\sigma_{0}>0$, le problème $\left(\mathbf{P}_{+}\right)$admet une solution unique dans $H_{\sigma_{0}}^{r_{0}^{-1}}\left(\mathbf{R}_{+}, \mathbf{H}^{1}\right.$ $\left.\left(\Omega_{+}\right)\right)$. En particulier, pour $r_{0}=1$, on a l'inégalité d'énergie:

$$
\int_{-\infty}^{+\infty} \mathrm{e}^{-2 \sigma t} E_{+}(u)(t) \mathrm{d} t \leqslant C\left(\sigma_{0}, \Gamma\right)|g|_{\sigma, 1,-1 / 2}^{2} \quad \forall \sigma \geqslant \sigma_{0}>0
$$

où $C\left(\sigma_{0}, \Gamma\right)$ désigne une constante générique dépendant de $\sigma_{0}$ et $\Gamma$, et $E_{+}$est l'énergie de u définie par:

$$
E_{+}(u)(t)=\int_{\Omega_{+}}\left(|\nabla u(t, x)|^{2}+\left|\frac{\partial u}{\partial t}(t, x)\right|^{2}\right) \mathrm{d} x .
$$

b) Le même résultat est valable pour le problème intérieur $\left(\mathbf{P}_{-}\right)$. La solution $u$ de $\left(\mathbf{P}_{+}\right) \cup\left(\mathbf{P}_{-}\right)$est alors représentable d'une façon unique par un potentiel retardé de double couche (2.1) sur $\Gamma$.

$L a$ densité $\varphi$ de ce potentiel, qui dépend linéairement de g, vérifie:

(2.10) $|\varphi|_{\sigma, r_{0}-1,1 / 2} \leqslant C\left(\sigma_{0}, \Gamma\right)|g|_{\sigma, r_{0},-1 / 2} \quad \forall \sigma \geqslant \sigma_{0}$.

On peut préciser que la dépendance en $\sigma_{0}$ des constantes $C\left(\sigma_{0}, \Gamma\right)$ dans (2.9) et (2.10) est de la forme $1 / \sigma_{0}^{2} \max \left(1 / \sigma_{0}^{2}, 1\right)$. Nous n'aurons pas besoin dans la suite de cette expression explicite des constantes. Gardons nous cependant en tête le fait que celles-ci ne restent pas bornées quand $\sigma_{0} \rightarrow 0$, ce qui correspond d'ailleurs à l'impossibilité bien connue de représenter la solution du problème de Helmholtz $\left(\mathbf{P}_{+}^{\omega}\right)$ par un potentiel de double couche quand $\omega$ est réel quelconque.

\subsection{Formulation variationnelle espace-temps pour l'équation en $\varphi$}

Occupons nous maintenant de l'équation (2.2) en $\varphi$. Les propriétés de l'opérateur $D$ sont de nouveau étudiées grâce à sa transformée de Laplace 
$D_{\omega}$. Rappelons pour cela les résultats suivants qui figurent implicitement dans [1].

Lemme 1 a) Pour $\operatorname{Im} \omega>0, D_{\omega}$ est un isomorphisme entre $\mathbf{H}^{1 / 2}(\Gamma)$ et $\mathbf{H}^{-1 / 2}(\Gamma)$. Sa norme vérifie la majoration:

(2.11) $\left\|D_{\omega}\right\| \leqslant C\left(\sigma_{0}, \Gamma\right)|\omega|^{2}$ pour tout $\operatorname{Im} \omega \geqslant \sigma_{0}>0$.

b) De plus, l'inégalité de coercivité suivante a lieu:

(2.12) $\operatorname{Re}\left\langle D_{\omega} f,-\mathrm{i} \omega f\right\rangle \geqslant C\left(\sigma_{0}, \Gamma\right)|f|_{1 / 2}^{2} \quad \forall f \in \mathbf{H}^{1 / 2}(\Gamma)$.

Puis par la formule de Parseval:

Théorème 2 a) $D$ est un opérateur linéaire continu de $H_{\sigma}^{2}\left(\mathbf{R}_{+}, \mathbf{H}^{1 / 2}(\Gamma)\right)$ dans $H_{\sigma}^{0}\left(\mathbf{R}_{+}, \mathbf{H}^{-1 / 2}(\Gamma)\right)$, dont la norme est majorée indépendamment de $\sigma \geqslant \sigma_{0}>0$. Autrement dit:

$$
\left\{\begin{array}{l}
|D \varphi|_{\sigma, 0,-1 / 2} \leqslant C\left(\sigma_{0}, \Gamma\right)|\varphi|_{\sigma, 2,1 / 2} \\
\forall \varphi \in H_{\sigma}^{2}\left(\mathbf{R}_{+}, \mathbf{H}^{1 / 2}(\Gamma)\right) \text { et } \sigma \geqslant \sigma_{0}>0
\end{array} .\right.
$$

b) On a l'inégalité de coercivité suivante:

$$
\left\{\begin{array}{l}
\int_{-\infty}^{+\infty} \mathrm{e}^{-2 \sigma t}\left\langle D \varphi(t, .), \varphi^{\prime}(t, .)\right\rangle \mathrm{d} t \geqslant C\left(\sigma_{0}, \Gamma\right)|\varphi|_{\sigma, 0,1 / 2}^{2} \\
\forall \varphi \in H_{\sigma}^{2}\left(\mathbf{R}_{+}, \mathbf{H}^{1 / 2}(\Gamma)\right), \quad \sigma \geqslant \sigma_{0}>0 .
\end{array}\right.
$$

Le crochet $\langle$,$\rangle désigne ici la dualité entre les espaces réels \mathbf{H}^{-1 / 2}(\Gamma)$ et $\mathbf{H}^{1 / 2}(\Gamma)$ tandis que dans (2.12) il s'agit de l'antidualité pour les espaces complexes correspondants.

L'inégalité (2.14) donne donc la formulation variationnelle suivante du problème (2.2):

Proposition 1 Si $g \in H_{\sigma}^{3}\left(\mathbf{R}_{+}, \mathrm{H}^{-1 / 2}(\Gamma)\right)$, la solution $\varphi$ de (2.2) est aussi l'unique solution du problème:

$$
\left\{\begin{array}{l}
\varphi \in H_{\sigma}^{2}\left(\mathbf{R}_{+}, \mathbf{H}^{+1 / 2}(\Gamma)\right) \text { tel que: } \\
\int_{-\infty}^{+\infty} \mathrm{e}^{-2 \sigma t}\left\langle D \varphi(t), \psi^{\prime}(t)\right\rangle \mathrm{d} t=\int_{-\infty}^{+\infty} \mathrm{e}^{-2 \sigma t}\left\langle g(t), \psi^{\prime}(t)\right\rangle \mathrm{d} t \\
\forall \psi \in H_{\sigma}^{2}\left(\mathbf{R}_{+}, \mathbf{H}^{1 / 2}(\Gamma)\right)
\end{array}\right.
$$

Il nous reste à traiter le problème de l'hyper-singularité de l'intégrale $D$. On revient encore sur $D_{\omega}$, pour lequel le même problème est maintenant bien maîtrisé (cf. [2], [3], [5]). La formule-clé est:

$$
\left\{\begin{aligned}
\left\langle D_{\omega} \varphi, \psi\right\rangle= & \frac{1}{4 \pi} \iint_{\Gamma \times \Gamma}\left\{\frac{-\omega^{2} v_{x} \cdot v_{y}}{|x-y|} \varphi(y) \bar{\psi}(x)\right. \\
& \left.+\frac{\overrightarrow{\operatorname{rot}}_{\Gamma} \varphi(y) \cdot \overrightarrow{\operatorname{rot}}_{\Gamma} \bar{\psi}(x)}{|x-y|}\right\} e^{i \omega|x-y|} \mathrm{d} \sigma_{x} \mathrm{~d} \sigma_{y}
\end{aligned}\right.
$$


où $\overrightarrow{\operatorname{rot}}_{\Gamma} \varphi$ est le rotationnel vecteur de surface d'une fonction $\varphi$ définie sur $\Gamma$. II peut être défini par:

$$
\overrightarrow{\operatorname{rot}}_{\Gamma} \varphi(x)=\nu_{x} \wedge \operatorname{grad} \varphi(x) \quad(x \in \Gamma)
$$

où $\tilde{\varphi}$ est défini dans un voisinage tubulaire de $\Gamma$ par:

$$
\bar{\varphi}\left(x+\alpha v_{x}\right)=\varphi(x) \text { pour } x \in \Gamma,|\alpha| \text { assez petit } .
$$

Si $\varphi=\hat{\varphi}(\omega, x)$ et $\psi=\hat{\psi}(\omega, x)$ sont des transformées de Laplace de $\varphi(t, x)$ et $\psi(t, x)$, on peut alors revenir à celles-ci par la transformation inverse. Plus précisément, multipliant les deux membres de (2.16) par $\mathrm{i} \bar{\omega}$ puis intégrant de $-\infty+\mathrm{i} \sigma \mathrm{a}+\infty+\mathrm{i} \sigma$, la formule de Parseval nous donne:

$$
\left\{\begin{array}{l}
b(\varphi, \psi)=\int_{-\infty}^{+\infty} \mathrm{e}^{-2 \sigma t} \mathrm{~d} t \iint_{\Gamma \times \Gamma}\left\{\frac{v_{x} \cdot v_{y}}{4 \pi|x-y|} \varphi^{\prime \prime}(t-|x-y|, y) \psi^{\prime}(t, x)\right. \\
\left.+\frac{\overrightarrow{\operatorname{rot}}_{\Gamma} \varphi\left(t-|x-y|, y \mid \cdot \overrightarrow{\operatorname{rot}}_{\Gamma} \psi^{\prime}(t, x)\right.}{4 \pi|x-y|}\right\} \mathrm{d} \sigma_{x} \mathrm{~d} \sigma_{y}
\end{array}\right.
$$

où $b(\varphi, \psi)$ désigne la forme bilinéaire au ler membre de (2.15).

\section{$\$ 3$ Discretisation du probleme variationnel (2.15)}

Nous suivons la même démarche que dans [1]. La différence essentielle vient de la forme bilinéaire. Il nous faut donc préciser la discrétisation de celle-ci. Nous découplons le temps et l'espace, et commençons par une discrétisation en espace par une méthode de Galerkin. Ceci donne le problème semi-discrétisé suivant:

$$
\left\{\begin{array}{l}
\varphi_{h} \in H_{\sigma}^{2}\left(\mathbf{R}_{+}, \mathbf{V}_{h}\right) \text { tel que } \\
b\left(\varphi_{h}, \psi_{h}\right)=\int_{-\infty}^{+\infty} \mathrm{e}^{-2 \sigma t}\left\langle g_{h}(t, .), \psi_{h}^{\prime}(t, .)\right\rangle \mathrm{d} t \\
\forall \psi_{h} \in H_{\sigma}^{2}\left(\mathbf{R}_{+}, V_{h}\right)
\end{array}\right.
$$

où $\mathbf{V}_{h}$ est un sous-espace de dimension finie de $\mathbf{H}^{1 / 2}(\Gamma)$, et $g_{h}$ une approximation de $g$.

On renvoie à [4] pour la construction de $\mathbf{V}_{h}$. Disons simplement que $\mathbf{V}_{h}$ est constitué de fonctions qui sont images sur $\Gamma$ de fonctions polynômiales par morceaux, de degré $m_{1} \geqslant 1$, définis sur des domaines triangulaires de $\mathbf{R}^{2}$ de taille $h$. Nous ne discuterons pas non plus le remplacement de $(I)$ par une surface approchée $\left(\Gamma_{h}\right)$, qui introduit des erreurs dominantes, celles dûes à l'approximation de $\mathbf{H}^{1 / 2}(\Gamma)$ par $\mathbf{V}_{h}$. Voir le $\S 6$ de [1]. Au point de vue de la marche des calculs, rien ne change cependant. s'écrire:

Soit donc $\left(\varphi_{h}^{j}\right)_{1 \leqslant j \leqslant N_{h}}$ une base de $\mathbf{V}_{h}$, Comme $\varphi_{h} \in H_{\sigma}^{2}\left(\mathbf{R}_{+}, \mathbf{V}_{h}\right)$ peut

$$
\varphi_{h}(t, x)=\sum_{j=1}^{N_{h}} \alpha_{j}(t) \varphi_{h}^{j}(x)
$$


avec $\alpha_{j} \in H_{\sigma}^{2}\left(\boldsymbol{R}_{+}, \mathbf{R}\right)$, on peut écrire $b\left(\varphi_{h}, \psi_{h}\right)$ sous la forme:

$$
\begin{aligned}
b\left(\varphi_{h}, \psi_{h}\right)= & \sum_{j, l} \int_{-\infty}^{+\infty} e^{-2 \sigma t} \beta_{j}^{\prime}(t) \iint_{\Gamma \times \Gamma}\left[K_{j, l}^{1}(x, y) \alpha_{l}^{\prime \prime}(t-|x-y|)\right. \\
& \left.+K_{j, l}^{2}(x, y) \alpha_{l}(t-|x-y|)\right] \mathrm{d} \sigma_{x} \mathrm{~d} \sigma_{y} \mathrm{~d} t
\end{aligned}
$$

où, pour simplifier les écritures, on a posé:

$$
\begin{aligned}
& K_{j, l}^{1}(x, y)=\frac{v_{x} \cdot v_{y}}{4 \pi|x-y|} \varphi_{h}^{l}(y) \varphi_{h}^{j}(x) \\
& K_{j, l}^{2}(x, y)=\frac{\overrightarrow{\operatorname{rot}_{\Gamma}} \varphi_{h}^{l}(y) \cdot \overrightarrow{\operatorname{rot}}_{\Gamma} \varphi_{h}^{j}(x)}{4 \pi|x-y|} .
\end{aligned}
$$

On approxime ensuite les fonctions de $H_{\sigma}^{2}\left(\mathrm{R}_{+}, \mathrm{R}\right)$ par celles du sous-espace $H^{m_{2}}(\Delta t ; \mathbf{R})$ constitué de fonctions polynômiales de degré $m_{2} \geqslant 2$ dans chaque intervalle $\mathcal{J}_{n}=(n \Delta t,(n+1) \Delta t)$.

Posant alors

$$
\varphi_{h, \Delta t}(t, x)=\sum_{j} \alpha_{j, \Delta t}(t) \varphi_{h}^{j}(x),
$$

le problème discrétisé de (2.15) consiste à chercher:

$$
\left\{\begin{array}{l}
\alpha_{l, \Delta t} \in H_{\sigma}^{m_{2}}(\Delta t ; \mathbf{R}), l=1, \ldots, N_{h} \text { telles que } \\
\sum_{l} \int_{-\infty}^{+\infty} e^{-2 \sigma t} \beta_{j, \Delta t}^{\prime}(t) \iint_{\Gamma \times \Gamma}\left[K_{j, l}^{1}(x, y) \alpha_{l, \Delta t}^{\prime \prime}(t-|x-y|)\right. \\
\left.\quad+K_{j, l}^{2}(x, y) \alpha_{l, \Delta t}(t-|x-y|)\right] \mathrm{d} \sigma_{x} \mathrm{~d} \sigma_{y} \mathrm{~d} t \\
=\int_{-\infty}^{+\infty} e^{-2 \sigma t} \beta_{j, \Delta t}^{\prime}(t) \int_{\Gamma} g_{h, \Delta t}(t, x) \varphi_{h}^{j}(x) \mathrm{d} \sigma_{x} \mathrm{~d} t \\
\forall \beta_{j, \Delta t} \in H_{\sigma}^{m_{2}}(\Delta t ; \mathbf{R}), 1 \leqslant j \leqslant N_{h} .
\end{array}\right.
$$

Nous détaillons ici les calculs dans le cas $m_{2}=2$, pour montrer qu'avec un choix convenable de fonctions de base $\beta_{j, \Delta t},(3.2)$ permet effectivement de calculer les $\alpha_{l, \Delta t}$, c'est-à-dire finalement la fonction approchée $\varphi_{h, \Delta t}$. La généralisation à $m_{2}$ quelconque est, comme dans [1], simple dans le principe mais plus longue à écrire. simple donne:

Dans ce cas, $\alpha_{l, \Delta t}^{\prime \prime}$ est une constante dans $\mathcal{A}_{n}$. Notons la $a_{l}^{n}$, et un calcul

$$
\text { (3.3) }\left\{\begin{array}{l}
\alpha_{l, \Delta t}(t)=\frac{1}{2}\left(t-t_{n}\right)^{2} a_{l}^{n}+\Delta t \sum_{m=0}^{n-1}\left(t-t_{m}+\frac{1}{2} \Delta t\right) a_{l}^{m} \\
t \in \mathcal{J}_{n} .
\end{array}\right.
$$

Prenant alors:

$$
\beta_{j, \Delta t}^{\prime}(t)=\left(\beta_{j, \Delta t}^{n}\right)^{\prime}(t)=\left\{\begin{array} { l } 
{ 0 } \\
{ t - t _ { n - 1 } } \\
{ t _ { n + 1 } - t }
\end{array} \left\{\begin{array}{l}
t \leqslant t_{n-1} \text { ou } t \geqslant t_{n+1} \\
t_{n-1}<t<t_{n} \\
t_{n}<t<t_{n+1}
\end{array}\right.\right.
$$


et substituant (3.3), (3.4) dans (3.2) on obtient un système de la forme:

$$
\sum_{k=0}^{n} M^{(n-k)} A^{k}=B^{n}
$$

où, au ler membre, $A^{k}$ est le vecteur $\left(a_{1}^{k}, \ldots, a_{N_{k}}^{k}\right)^{t}$ et les matrices $M^{(n-k)}$, ne dépendant que de $n-k$, sont nulles pour $n-k>\left[\frac{\mathrm{d}(I)}{\Delta t}\right] ; \mathrm{d}(\Gamma)$ désigne le diamètre de $\Gamma$. Pour obtenir (3.5), la même technique de décomposition des intégrales doubles, déjà exposée dans [1], est réutilisée. Aussi, nous nous contentons de vérifier que pour $\Delta t$ assez petit, la matrice $M^{0}$ est inversible.

On a:

$$
\begin{aligned}
M_{j, l}^{0}= & \int_{t_{n}}^{t_{n+1}} \mathrm{e}^{-2 \sigma t}\left(t_{n+1}-t\right) \iiint_{\left\{x-y \mid<t-t_{n}\right\}}\left[K_{j, l}^{1}(x, y)\right. \\
& \left.+\frac{1}{2}\left(t-|x-y|-t_{n}\right)^{2} K_{j, l}^{2}(x, y)\right] \mathrm{d} \sigma_{x} \mathrm{~d} \sigma_{y} \mathrm{~d} t
\end{aligned}
$$

où, après le changement de variables $s=t-t_{n}$ et simplifiant par le facteur $\mathrm{e}^{-2 \sigma t_{n}}$ qui existe dans tous les termes aux deux membres de (3.5), et conservant la notation $M^{0}$ pour $\mathrm{e}^{-2 \sigma t_{n}} M^{0}$ :

$$
\begin{aligned}
M_{j, l}^{0}= & \int_{0}^{\Delta t} \mathrm{e}^{-2 \sigma t}(\Delta t-s) \iiint_{\{|x-y|<s\}}\left[K_{j, l}^{1}(x, y)\right. \\
& \left.+\frac{1}{2}(s-|x-y|)^{2} K_{j, l}^{2}(x, y)\right] \mathrm{d} \sigma_{x} \mathrm{~d} \sigma_{y} \mathrm{~d} t
\end{aligned}
$$

on a le

Lemme 2 La matrice $M^{0}$ est symétrique, définie positive.

Démonstration. Il est clair d'après les expressions de $K_{j, l}^{1}$ et $K_{j, l}^{2}$ que $M^{0}$ est symétrique. Soit en suite $X=\left(x_{1}, \ldots, x_{N_{h}}\right) \in \mathrm{R}^{N_{h}}$, et posant $X_{h}=\sum_{j} x_{j} \varphi_{h}^{j} \in V_{h}$,
on a:

$$
{ }^{t} X M^{0} X=\int_{0}^{\Delta t} \mathrm{e}^{-2 \sigma s}(\Delta t-s) I(s) \mathrm{d} s
$$

avec $I(s)=I_{1}(s)+I_{2}(s)$ et

$$
\begin{aligned}
& I_{1}(s)=\iiint_{\{|x-y|<s\}} \frac{v_{x} \cdot v_{y}}{4 \pi|x-y|} X_{h}(x) X_{h}(y) \mathrm{d} \sigma_{x} \mathrm{~d} \sigma_{y} \\
& I_{2}(s)=\iiint_{\{|x-y|<s\}} \frac{\overrightarrow{\operatorname{rot}}_{\Gamma} X_{h}(x) \cdot \overrightarrow{\operatorname{rot}}_{\Gamma} X_{h}(y)}{4 \pi|x-y|} \mathrm{d} \sigma_{x} \mathrm{~d} \sigma_{y} .
\end{aligned}
$$


Considérons la lère intégrale:

$$
I_{1}(s)=\int_{\Gamma} \nu_{x} X_{h}(x) \mathrm{d} \sigma_{x} \cdot \int_{\left\{y \in \Gamma_{i}|x-y|<s ;\right.} \frac{v_{y} X_{h}(y)}{4 \pi|x-y|} \mathrm{d} \sigma_{y}
$$

pour $s<\Delta t$ suffisamment petit, on peut assimiler l'ensemble $\{y \in \Gamma ;|x-y|<s\}$ au disque de centre $x$, de rayon $s$ dans le plan tangent à $\Gamma$ en $x$, ce qui donne:

$$
\int_{\{y \in \Gamma ;|x-y|<s ;} \frac{v_{y} \cdot X_{h}(y)}{4 \pi|x-y|} \mathrm{d} \sigma_{y}=\frac{s}{2} v_{x} X_{h}(x)
$$

par suite, pour $s$ assez petit

$$
I_{1}(s) \geqslant c_{1} s\left|X_{h}\right|_{L^{2}(n)}^{2} .
$$

De même

$$
I_{2}(s) \geqslant c_{2} \cdot s^{3}\left|\overrightarrow{\operatorname{rot}}_{l}-X_{h}\right|_{L^{2}(\Omega)}^{2} .
$$

En intégrant, on obtient le résultat.

Remarques. $1^{0} /$ Comme $\left|\overrightarrow{\operatorname{rot}}_{\Gamma} X_{h}\right|_{L^{2}(\Omega)}=0\left(\frac{1}{h}\right)\left|X_{h}\right|_{L^{2}(n)}$ (cf. [4]), les deux termes de $I(s)$ sont en fait de même ordre si $\Delta t / h$ reste borné.

$2^{0} /$ On peut donner un argument fonctionnel à ces calculs approchés avec le lemme suivant:

Lemme 3 Posons pour $f \in L^{2}(\Gamma)$,

$$
f_{s} f(x)=\frac{1}{s} \int_{\{y \in \Gamma ;|y-x|<s \mid} \frac{f(y)}{|x-y|} \mathrm{d} \sigma_{y} .
$$

Alors il existe une constante $C>0$ telle que

$$
\left|g_{s} f\right|_{\mathbf{L}^{2}(I)} \leqslant C|f|_{\mathbf{L}^{2}(\Omega)}
$$

et quand $s \rightarrow 0, \Im_{s} f \rightarrow f$ dans $\mathrm{L}^{2}(\Gamma)$.

Démonstration. On peut écrire d'après l'inégalité de Cauchy-Schwarz:

$$
\left|f_{s} f(x)\right|^{2}<\frac{1}{s^{2}}\left(\int_{\Gamma} \frac{|f(y)|^{2}}{|x-y|} 1_{\{|x-y|<s\}} \mathrm{d} \sigma_{y}\right)\left(\int_{\Gamma}^{1} \frac{\{|x-y|<s\}}{|x-y|} \mathrm{d} \sigma_{y}\right)
$$

la dernière intégrale est majorée indépendamment de $x$ par un $0(s)$, par suite

$$
\begin{aligned}
\left|g_{s} f\right|_{L^{2}(I)}^{2} & \leqslant \frac{0(s)}{s^{2}} \int_{\Gamma} \int_{\Gamma} \frac{|f(y)|^{2}}{|x-y|} 1_{|| x-y \mid<s\}} \mathrm{d} \sigma_{y} \\
& \leqslant \frac{0(s)}{s^{2}} \int_{\Gamma}|f(y)|^{2} \mathrm{~d} \sigma_{y} \int_{\{x,|x-y|<s\}} \frac{\mathrm{d} \sigma_{x}}{|x-y|}
\end{aligned}
$$

d'où (3.7). 
Il est d'autre part clair que pour $f$ régulière, $\oint_{s} f(x) \rightarrow f(x)$ pour tout $x \in \Gamma$, et $\left|\mathscr{l}_{s} f(x)\right| \leqslant \max _{\Gamma}|f(x)| \cdot O(s)$, par suite $\oiint_{s} f \rightarrow f$ dans $\mathbf{L}^{2}(\Gamma)$ pour ces fonctions. Il ne reste plus qu'à passer par le densité dans $\mathrm{L}^{2}(\Gamma)$ des fonctions régulières pour conclure.

L'application de ce lemme dans $I_{1}(s)$ est immédiate. Ainsi, la relation (3.5) constitue bien une équation linéaire permettant de calculer de proche en proche les valeurs $\operatorname{des} A^{k}$.

\section{$\$ 4 \quad$ Resultat de stabilite et de convergence}

Les principes de ce schéma ressemblent en tous points à ceux déjà analysés dans le cas du problème de Dirichlet résolu avec un potentiel de simple couche [1]. Aussi nous nous contentons d'énoncer ci-dessous les résultats obtenus.

Théorème 3 a) (Stabilité). Si $g_{h, \Delta t}$ est une approximation consistante de $g$ dans $H_{\sigma}^{1}\left(\boldsymbol{R}_{+}, \mathbf{H}^{-1 / 2}(\Gamma)\right)$, le schéma proposé au $\$ 3$ est stable au sens suivant:

(4.1) $\left|\varphi_{h, \Delta t}\right|_{\sigma, 0,1 / 2} \leqslant$ constante

quand $h, \Delta t \rightarrow 0$.

b) (Estimation d'erreurs). Si g est suffisamment régulière, le schéma converge quand $m_{1} \geqslant 1$ et $m_{2} \geqslant 3$ et l'on a l'estimation suivante:

$$
\begin{aligned}
\left|\varphi-\varphi_{h, \Delta t}\right|_{\sigma, 0,1 / 2} \leqslant & C\left\{\left|g-g_{h, \Delta t}\right|_{\sigma, 1,-1 / 2}+\frac{h^{m_{1}+1 / 2}}{\Delta t}|\varphi|_{\sigma, 2, m_{1}+1}\right. \\
& \left.+\Delta t^{m_{2}-2}|\varphi|_{\sigma, m_{2}+1,1 / 2}\right\}
\end{aligned}
$$

Enfin, si $\varphi_{h, \Delta t}$ est utilisé à la place de $\varphi$ dans (2.1) pour calculer $u$, nous obtenons une solution approchée $u_{h, \Delta r}$ du probème $\left(\mathbf{P}_{+}\right)$dont l'écart avec la solution exacte est estimée dans le:

Thèorème 4 Dans les conditions du Théorème 3, on $a$ :

$$
\left\{\begin{array}{l}
\int_{0}^{\infty} \mathrm{e}^{-2 \sigma t} E\left(u-u_{h, \Delta t}\right)(t) \mathrm{d} t \leqslant C\left|g-g_{h, \Delta t}\right|_{\sigma, 1,-1 / 2}\left|\varphi-\varphi_{h, \Delta t}\right|_{\sigma, 0,1 / 2} \\
\text { où }\left|\varphi-\varphi_{h, \Delta t}\right|_{\sigma, 0,1 / 2} \text { est majoré par (4.2). }
\end{array}\right.
$$

\section{Conclusion}

Nous avons proposé une formulation variationnelle espace-temps pour le calcul d'une onde acoustique diffractée par une surface rigide, utilisant un potentiel retardé de double couche. Cette formulation conduit aussi après une discrétisation de type éléments finis, à un schéma de calculs des valeurs de la 
densité du potentiel, de proche en proche en suivant la marche du temps, et ne faisant pas intervenir des intégrales hyper-singulières. De plus, les calculs algébriques du schéma sont quasiment explicites, en ce sens qu'ils n'exigent qu'une seule inversion de matrice, qui est de plus définie, positive et creuse. C'est donc une situation analogue aux calculs utilisant la formule de Kirchoff. L'avantage (purement théorique pour le moment car l'implantation numérique de notre schéma reste à faire) est qu'ici des résultats de stabilité et de convergence sont obtenus, grâce à une propriété de coercivité de la formule variationnelle.

\title{
Bibliographie
}

[1] Bamberger, A.; Ha Duong, T.: Formulation Variationnelie Espace-Temps pour le Calcul par potentiel Retardé de la Diffraction d'une onde acoustic (I). Math. Meth. in the Appl. Sci. 8 (1986) $405-435$

[2] Bendali, A.: Approximation par èléments finis de surface de problèmes de diffraction des ondes électromagnétiques. Thèse d'Etat, Université Pierre et Marie Curie 1984

[3] Hamdi, M. A.: Une formulation variationnelle par équations intégrales pour la résolution de l'équation de Heimholtz avec des conditions aux limites mixtes. CRAS, série II, 292 (1981) 17-20

[4] Nedelec, J. C.: Approximation des équations intégrales en mécanique et en physique. Cours de l'Ecole d'Eté d'Analyse Numérique EDF-CEA-INRIA 1977

[5] Nedelec, J. C.: Approximation par potentiel de double couche du problème de Neumann extérieur. CRAS, série A, 286 (1977) 616-619

\author{
A. Bamberger \\ T. Ha Duong \\ Ecole Polytechnique \\ Centre de Mathèmatiques Appliquees \\ F-91128 Palaiseau Cedex
}

